\title{
Modulation of induced gamma band activity in the human EEG by attention and visual information processing
}

\author{
Matthias M. Müller ${ }^{\mathrm{a}, *}$, Thomas Gruber ${ }^{\mathrm{a}}$, Andreas Keil ${ }^{\mathrm{b}}$ \\ ${ }^{a}$ Cognitive Neuroscience, Department of Psychology, University of Liverpool, Eleanor Rathbone Building, \\ Liverpool L69 7ZA, UK \\ ${ }^{\mathrm{b}}$ Department of Psychology, University of Konstanz, Konstanz, Germany
}

\begin{abstract}
Here we present a series of four studies aimed to investigate the link between induced gamma band activity in the human EEG and visual information processing. We demonstrated and validated the modulation of spectral gamma band power by spatial selective visual attention. When subjects attended to a certain stimulus, spectral power was increased as compared to when the same stimulus was ignored. In addition, we showed a shift in spectral gamma band power increase to the contralateral hemisphere when subjects shifted their attention to one visual hemifield. The following study investigated induced gamma band activity and the perception of a Gestalt. Ambiguous rotating figures were used to operationalize the law of good figure (gute Gestalt). We found increased gamma band power at posterior electrode sites when subjects perceived an object. In the last experiment we demonstrated a differential hemispheric gamma band activation when subjects were confronted with emotional pictures. Results of the present experiments in combination with other studies presented in this volume are supportive for the notion that induced gamma band activity in the human EEG is closely related to visual information processing and attentional perceptual mechanisms. (c) 2000 Published by Elsevier Science B.V. All rights reserved.
\end{abstract}

Keywords: Induced gamma band response; Human EEG; Visual information processing; Attention; Gestalt

\footnotetext{
* Corresponding author.

E-mail address: m.mueller@liverpool.ac.uk (M.M. Müller).
} 


\section{Introduction}

Oscillatory cortical activities in the human brain above $20 \mathrm{~Hz}$, i.e. in the gamma band, do not form a homogeneous class of responses. Experimental evidence demonstrates diversity with respect to both perceptual and neurophysiological mechanisms. In 1992, Galambos introduced the following classification of EEG responses with power in the gamma band (Galambos, 1992):

1. Spontaneous gamma rhythms. Such rhythms appear without obvious relation to an external event.

2. Evoked gamma band responses. These are event-related responses elicited by and precisely time-locked to an external stimulus. In expansion to the classification by Galambos, one should further divide this category into (a) transient evoked gamma band responses and (b) driven responses like the steady-state auditory or visual evoked response.

3. Emitted gamma band oscillations. Gamma band activity time-locked to a stimulus that has been omitted. Conceptually, but not technically, these may be subsumed under category 2.

4. Induced gamma band rhythms are initiated by an event but are not time- and phaselocked to the eliciting stimulus. This category in itself encompasses a variety of different processes.

In accordance with the topic of this volume, we focus on induced gamma band responses in the human brain. A series of hypotheses seeks to attribute meaning to oscillating cell assemblies. One of the earlier, empirically based hypothesis was put forward by Walter Freeman. According to Freeman (1975, see also Freeman, 1996; Freeman and van Dijk, 1987; Freeman and di Prisco, 1986) the stimulus itself is coded in oscillatory patterns or, more precisely, as a dynamic state of a non-linear system that appears to the observer as oscillations. On the basis of intracortical recordings from the olfactory bulb of the rabbit, Freeman and di Prisco (1986) concluded that after the presentation of a learned odor, the system switches from a spatially and temporally unpatterned chaotic state to a global odor-specific state which is characterized by a single near-limit attractor. In other words, the attractor governing the dynamic pattern is related to stimulus encoding, everything that is not coherent to this signal is dismissed as noise.

The most popular hypothesis that in fact could be a consequence of Freeman's model, the temporal binding hypothesis, predicts that synchronized oscillatory neural activity is the mechanism by which various brain regions form one percept and are, therefore, the key mechanism for feature binding (Abeles, 1982; Eckhorn et al., 1990; Gray et al., 1989, 1990; Gray and Singer, 1987; Malsburg and Schneider, 1986; Milner, 1974; Singer and Gray, 1995). Thus, oscillations in the gamma band range were considered to be functionally different from oscillations in the alpha range. It was hypothesized that alpha band oscillations reflect idling in neural mass systems (Hari and Salmelin, 1997; Pfurtscheller, 1992; Pfurtscheller and Aranibar, 1979; Pfurtscheller et al., 1993; Pfurtscheller and Klimesch, 1992). This idling state would allow the system to start more rapidly than by a 'cold start' (Hari and Salmelin, 1997).

In the last decade, most of the studies that investigated induced gamma band responses were conducted in animal research (see Singer and Gray, 1995 for an overview and Engel et al. this volume). One of the early animal experiments has demonstrated that long-range synchronization in area 17 of the anaesthetized cat reflects global stimulus properties (Gray and Singer, 1989). In this experiment, multiunit activity was recorded from two sites which preferred vertical orientations and were separated by $7 \mathrm{~mm}$. The corresponding receptive fields were non-overlapping and co-linearly arranged. This arrangement allowed three different stimulus conditions: (a) a long continuous light bar moving across both fields; (b) two independent light bars moving in the same direction; and (c) the same two bars moving in opposite directions. The results showed that the induced gamma band responses of the two receptive fields were synchronized when the long bar was presented. In the case of the two independent bars moving in the same direction, 
the synchronization across the two receptive fields became weaker and totally disappeared if the motion of the stimuli was incoherent. These results gave raise to the notion that induced gamma band activity is related to the features of the stimuli and thus were considered to support the temporal binding hypothesis.

In one series of experiments on induced gamma band activity in the human EEG (Müller et al., 1996), we aimed at mimicking the work on animals, e.g. the Gray et al. (1989) study, as closely as possible. We used the same stimulus configuration consisting of a long coherently moving bar and two small bars moving in opposite directions. We hypothesized that if synchronized neural activity is related to cortical object representation, we should see enhanced power in the gamma band when subjects processed the long bar. In the condition where two incoherently moving bars were presented, we expected a marked reduction in gamma band power as compared to the coherently moving long bar since - on the basis of a simplified model - two cell assemblies with no phase coherence should code the two bars. In the macroscopic EEG recording, a reduction in gamma power would be the consequence. In accordance with our hypothesis, we observed significantly increased gamma band power on posterior electrode sites when subjects attended the long bar as compared to the condition during which subjects attended to the two bars moving in opposite directions. This finding was replicated in a subsequent follow-up study (Müller et al., 1997a,b).

There are now a series of reports that confirm a modification of human EEG gamma band power as a function of stimulus properties. Lutzenberger et al. (1995) found increased gamma band power when lines in a visual quarter field moved coherently, giving the impression of a waterfall, as compared to a condition during which the lines were moving randomly. In a series of experiments Tallon-Baudry and colleagues reported a link between stimulus features and the representation of a stimulus and induced gamma band activity (Tallon et al., 1995; Tallon-Baudry and Bertrand, 1999; Tallon-Baudry et al., 1996, 1997a,b). In addition, these authors have pointed out a possible link between gamma band activity and memory processes (Tallon-Baudry et al., 1998). The experiments by the Lyon group are fully described elsewhere in this volume, we refer the reader to that chapter. In using illusionary triangles and squares, Herrmann et al. (1999) have replicated the findings of Tallon-Baudry and colleagues but only with respect to evoked gamma band responses. The work is also fully described elsewhere in this volume.

Recently, Rodriguez et al. (1999) have shown an increase in gamma band power when subjects were confronted with so-called Mooney faces (black/white shapes of faces) as compared to when these figures were presented upside-down, which did not allow subjects to identify a face. In addition to the studies mentioned so far, induced gamma band responses in humans have been reported in the auditory cortex (Jokeit and Makeig, 1994; Tallon-Baudry and Bertrand, 1999), in the sensorimotor cortex (Kristeva-Feige et al., 1993), during the processing of words in contrast to pseudo words (Eulitz et al., 1996; Lutzenberger et al., 1994; Pulvermüller et al., 1996, 1995) and in a classical conditioning paradigm (Miltner et al., 1999).

In the following, we present a series of four experimental studies. Three of them (studies 2, 3 and 4) have been published previously (Gruber et al., 1999; Keil et al., 1999; Müller et al., 1999).

The first two experiments were conducted to demonstrate and validate a link between the modulation of induced gamma band activity and spatial selective visual attention. In previous research, it has been suggested that attended information within the visual field, or - to express it with a widely used metaphor - information which falls within the attentional 'spotlight' elicits larger sensory-evoked responses as compared to the information outside the 'spotlight' (Hillyard and Anllo-Vento, 1998; LaBerge, 1995; Mangun, 1995; Posner and Petersen, 1990; Posner and Dehaene, 1994). Such an amplification mechanism has been demonstrated in EEG recordings by showing an amplitude augmentation of particular components of the visual evoked potential (VEP) when a stimulus was attended as compared to when the stimulus was ignored (Anllo-Vento and Hillyard, 
1996; Hillyard and Anllo-Vento, 1998; Hillyard et al., 1998; Luck and Ford, 1998; Mangun, 1995). It has been validated using positron emission tomography (PET); blood flow was increased in those regions of the visual cortex that were related to the sensory processing of an attended stimulus (Corbetta et al., 1993, 1995; Heinze et al., 1994; Mangun et al., 1997). Recently, these findings were confirmed by means of functional magnetic resonance imaging (fMRI; Beauchamp et al., 1997; Haug et al., 1998; Martinez et al., 1999). In sum, experimental results support the idea that visual information in attended sensory pathways triggers an amplification, i.e. stronger neuronal responses as compared to unattended pathways, resulting in a larger signal. Consequently, if induced gamma band activity is related to visual information processing, EEG spectral gamma power should be enhanced when subjects attend to a certain stimulus as compared to when subjects ignore that stimulus. The experimental outcome presented in Section 2 confirms this hypothesis.

The next study demonstrates a link between the perception of a Gestalt and induced gamma band activity. A traditional approach used to study visual perception exploits the properties of ambiguous (bistable) figures (Attneave, 1971; Kanizsa and Luccio, 1995). We used an ambiguous figure that, when rotated, biased the subjects' perception to that of either a sad or happy face. On the basis of the law of good figure (gute Gestalt), we assumed that induced gamma band responses will be associated with the perception of the sad and happy face rather than the continuous visual input.

In the last experimental study presented here, we investigated whether processing emotional pictures modulates gamma band activity differentially depending on the valence of the stimulus. Theoretically, affect and emotion in humans have been related to the integrated activity of brain circuits including structures such as the amygdala, hippocampus or anterior cingulate in addition to the neocortex particularly sensory representational zones (Davidson and Hugdahl, 1995; Derryberry and Tucker, 1992; LeDoux, 1995b; Liotti and Tucker, 1995; Tucker and Dawson, 1984). In particular, the prefrontal cortex has been suggested to be an important part of such a widespread affective network (Damasio, 1995; Davidson, 1992; Davidson et al., 1990). Based on the findings reported so far, it seems reasonable to predict that neural activity subserving affective processing might use high-frequency oscillatory coding in the relevant circuits as a means to integrate the activity of distributed structures.

\section{Attentional modulation of induced gamma band activity}

The following two studies were conducted to investigate whether EEG induced gamma band activity is modulated by visual selective spatial attention. As mentioned above, a consistent finding is an amplitude augmentation of certain components of the VEP when a stimulus is attended. This is seen as an attentional cortical facilitation in visual areas corresponding to the processing of the stimulus (Hillyard and Anllo-Vento, 1998; Hillyard et al., 1998). This facilitation mechanism should also apply for cell assemblies distributed across different functional visual areas or pathways and thus should result in an increase in spectral gamma power when attending to a certain stimulus as compared to ignoring that stimulus.

\subsection{Study 1}

In this study we instructed our subjects to attend to either a moving long bar presented in the right or left visual hemifield or to ignore the moving bar and attend a central fixation rectangle while performing a visual selective attention task related to the to-be attended location. As a baseline measure, the bar was presented as a standing bar for $600 \mathrm{~ms}$. After that the bar started to move with a speed of $2.45^{\circ} / \mathrm{s}$ for $1578 \mathrm{~ms}$ and disappeared for $900 \mathrm{~ms}$ while the fixation rectangle was still on the screen. With motion onset, the bar and the fixation rectangle changed colors and subjects had to detect and silently count the occurrence of a certain target color in the to-beattended location. Fig. 1 displays a schematic 


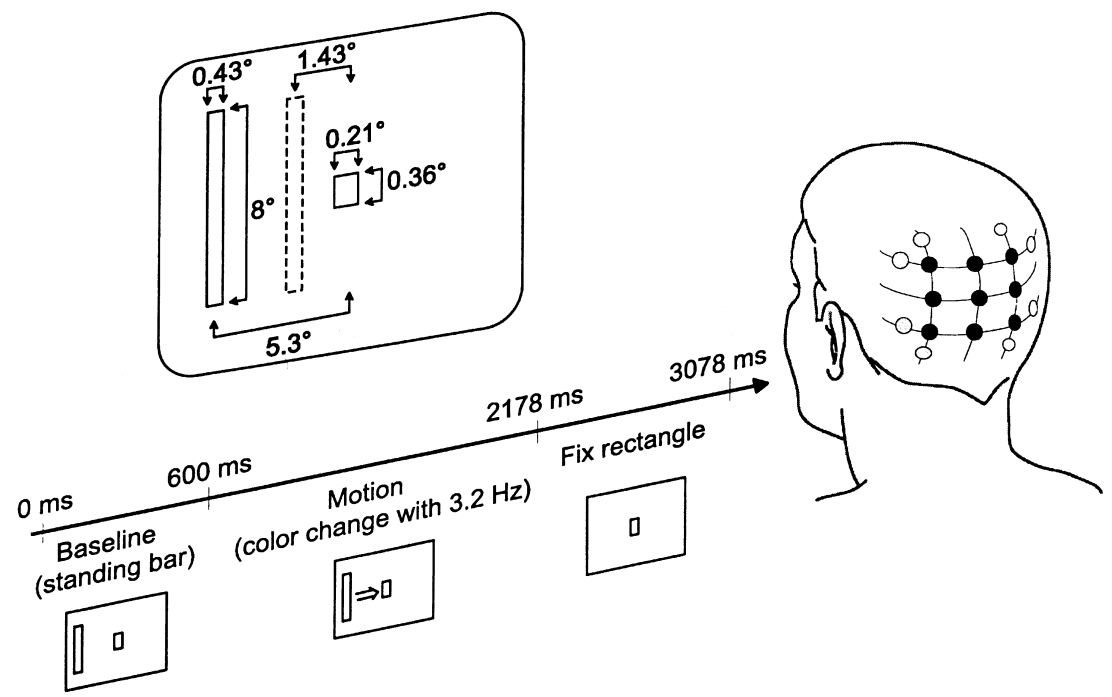

Fig. 1. Schematic representation of electrode locations and experimental setup. See text for detailed information.

representation of the stimulus configuration. The four experimental conditions (attend/unattend bar, left/right visual hemifield) were presented as a block of 200 trials each.

EEG was recorded by a $3 \times 3$ square grid $(3.5$ $\mathrm{cm}$ ) with the central electrode located $6 \mathrm{~cm}$ anterior to the inion to analyze cortical electrical activity over the occipito-parietal lobes (Lutzenberger et al., 1995; Maier et al., 1987). Since we calculated the current source density (CSD; Perrin et al., 1989) before transformation into the frequency domain, additional electrodes placed above and below the first and the third column, as well as to the right and the left of the first and third row were required (see Fig. 1). In addition, vertical and horizontal eye movements were monitored.

The evolutionary spectrum (Priestley, 1988) was calculated by means of the Discrete Gabor Transform (Qian and Chen, 1993) for single artifact free trials to determine time changes in the intensity of oscillatory activity. The frequency by time matrices of the single trails were averaged for further analysis (for more detailed information on this analysis procedure we refer the interested reader to Müller, 1998; Müller et al., 1996, 1997a). For statistical analysis six frequency bands for the gamma range 18-33, 33-49, 49-64, 65-80, 81-96,
96-111 $\mathrm{Hz}$ and the alpha band in the frequency range from 6 to $14 \mathrm{~Hz}$ were extracted. Each frequency band was divided by the respective spectral power of the baseline period in order to obtain the increase in spectral power relative to the baseline.

We found significantly enhanced power in the $65-80-\mathrm{Hz}\left(F_{1,7}=6.4, P<0.05\right)$ and in the 81-96$\mathrm{Hz}$ band $\left(F_{1,7}=8.6, P<0.05\right)$ when the bar was attended as compared to when attention was drawn away from the moving bar. In addition, no attention effect was present in the alpha band. For the following analysis we combined the two bands to a broader band expanding 65-96 Hz. The subsequent ANOVA revealed an enhancement of $67.7 \%$ of spectral power across stimulation sides and electrodes in the broad gamma band when attention was directed to the moving bar as compared to when attention was drawn away from the bar to the fixation rectangle $\left(F_{1,7}=10.7, P=0.01\right)$. However, no effect was found with respect to stimulation side or electrode column, which indicated that there was no statistically significant difference when the left or right visual hemifield was attended. Rather we saw a broad gamma band distribution over the posterior scalp surface area which was covered by the $3 \times 3$ electrode grid. 


\subsubsection{Summary and conclusion}

In this first experiment we found an increase of spectral gamma power when subjects attended to the moving bar as compared to when subjects ignored the bar. No such effect was present in the alpha band, indicating no difference in the general level of arousal (Ota et al., 1996). In this study, EEG was obtained only from occipital electrode sites. Therefore, the topography of the attention effect remained unknown.

\subsection{Study 2}

Study 2 was designed to overcome the limited EEG recording sites of study 1 by using a 128channel EEG-montage. Aside from an enhancement in spectral gamma power when the moving stimulus was attended, we investigated whether a shift in the topographical distribution of the gamma band response to the hemisphere contralateral to the to-be-attended side after the onset of the attention direction cue can be observed. Thus, the present study cannot only validate the effects found in study 1 , but also extend our knowledge to events in brain regions related to the visual input.

Subjects were confronted with a complex pattern of colored squares and rectangles presented on a computer screen (see Fig. 2). After $500 \mathrm{~ms}$ an arrow indicated whether attention had to be shifted to the left or the right half of the screen to detect target stimuli while maintaining gaze at a central fixation point (Fig. 2, middle panel). One second after arrow onset, the attended visual hemifield either moved horizontally with $2.6^{\circ} / \mathrm{s}$ for $2 \mathrm{~s}$ and the unattended half of the screen was motionless or vice versa (Fig. 2, right panel). Starting with motion onset, the squares and rectangles changed colors and subjects were instructed to detect and silently count the occurrence of a certain target color in one of the geometric figures. For each experimental condition (attended/unattended motion, left/right visual hemifield), 120 trials were presented in randomized order.

EEG was recorded continuously using an EGI (Electrical Geodesics Inc., Eugene, OR, USA) high density array with 128 electrodes. Electrode positions as projected to a plain are shown in Fig. 3.

For artifact rejection and artifact correction, single epochs were submitted to a procedure developed by Junghöfer et al. (2000; Statistical Correction of Artifacts in Dense Array Studies, SCADS). For further analysis the average reference was used. Extraction of induced gamma band power was performed using a Fast Fourier Transform (FFT) proposed by Feige, which is included in the avg-q software package (Feige, 1996). This approach computes the power spectrum in a manner similar to that described by Makeig (1993). In addition, it enables the user to employ a modular approach to spectral analysis. In the present work, the time-locked activity (i.e. the ERP) was sub- (a)

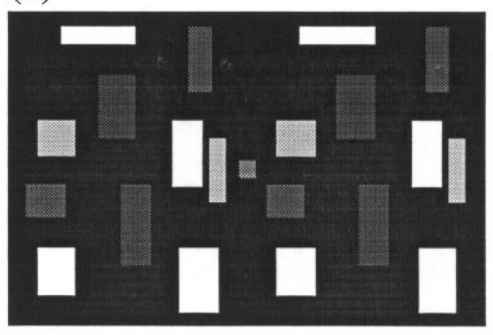

(b)

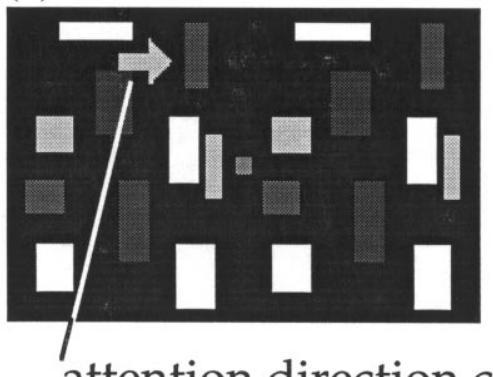

(c)

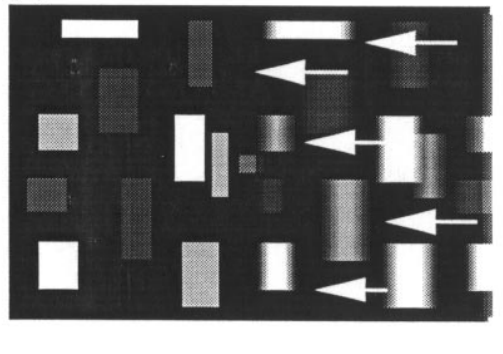

\section{attention direction cue}

Fig. 2. Schematic representation of one trial: (a), stimulus onset $(500 \mathrm{~ms})$; (b), arrow indicating whether the subject had to attend the left or the right half of the screen $(500 \mathrm{~ms})$; and (c), rotation of the left or right half of the screen (2000 ms). Note, between arrow offset and motion onset a further period of $500 \mathrm{~ms}$ without arrow was introduced. 


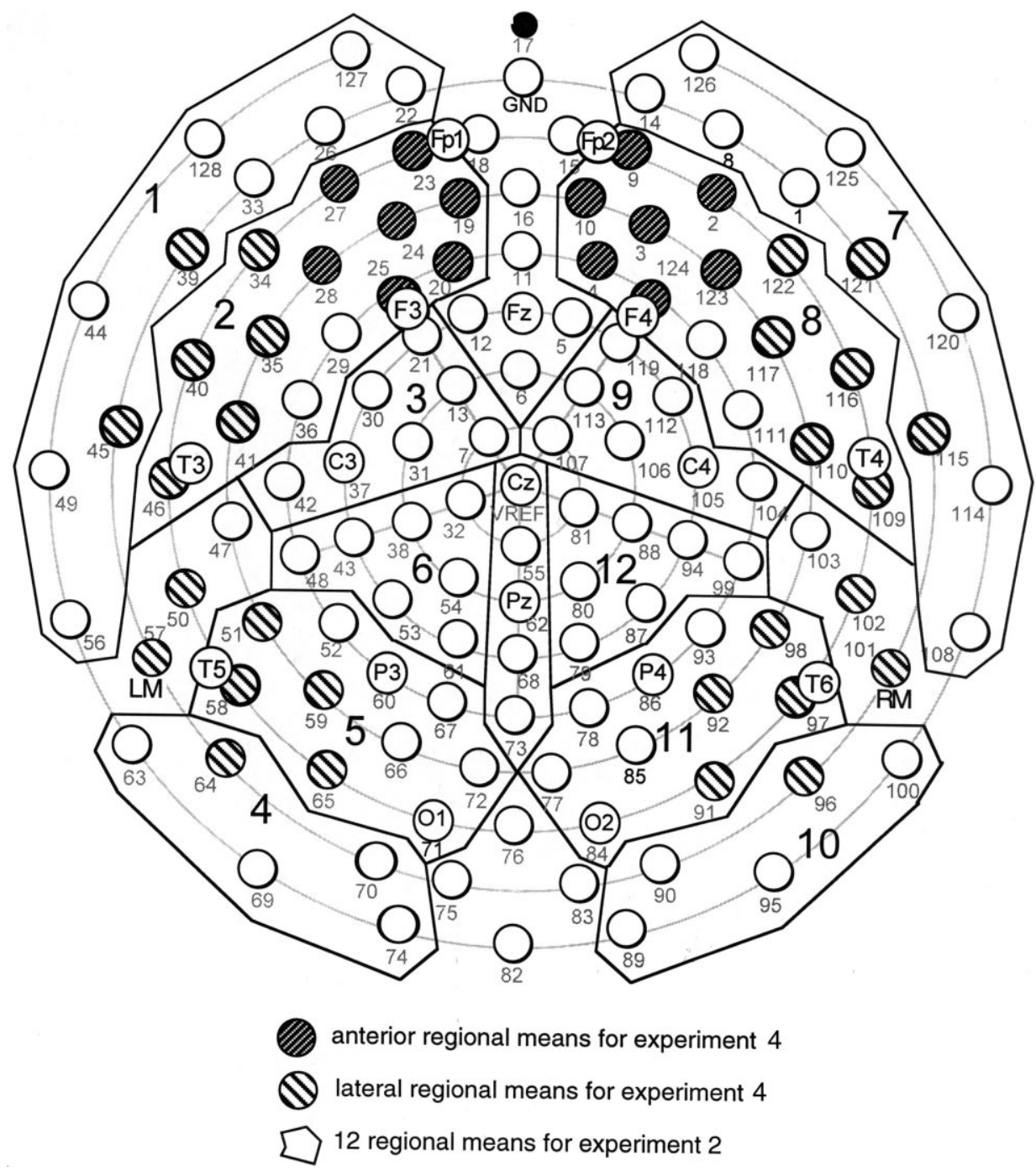

Fig. 3. Regional means resulting from the 128 channels montage, 10-20 electrode sites are given to allow for a comparison. Borders refer to regional means for study 2. Occipital electrode clusters for study 3 are equivalent for clusters 4, 5, 10 and 11 . Striped electrode locations refer to anterior and frontal clusters of study 2 .

tracted from the signal in a first step. Subsequently, the epochs were demeaned and detrended. A Welch-tapered analysis window was shifted across the recording epoch, thus determining the time resolution of the spectral estimate. The power spectrum for each of these analysis windows was determined using two FFT windows with a given overlap. The resulting power spectra were averaged across epochs (for a more detailed description of the analysis procedure see Gruber et al., 1999; Keil et al., 1999; Müller et al., 1999).
Four frequency bands were formed in the gamma band range by averaging the respective frequencies $(19-35,35-51,51-66$ and $66-82 \mathrm{~Hz}$ ) and a lower frequency range from 4 to $12 \mathrm{~Hz}$. Because of the on-line $100 \mathrm{~Hz}$ lowpass filter, higher frequencies were not analyzed. Each band was baseline corrected by dividing the mean spectral power by the respective baseline power (black screen). Thus, the normalized time windows represent the spectral power relative to the dark screen. Next, averaged and baseline corrected 
spectral power was normalized according to McCarthy and Wood (1985) and regional means were calculated on the basis of a suggestion by Dien et al. (1997; see Fig. 3). In addition, we were interested in whether or not the evoked spectral response shows effects similar to the induced response. For this analysis the evoked response was transformed into the frequency domain in a similar manner as described for the induced gamma band response, and identical statistical analyses were performed.

We found a significant power increase over the left and right parieto-occipital regional means (5 and 11 in Fig. 3) only in the frequency band from 35 to $51 \mathrm{~Hz}$ when the transposition was attended compared with the unattended transposition $\left(F_{1,10}=8.64, P=0.01\right)$. This increase was higher at parieto-occipital sites contralateral to the tobe-attended transposing screen and more so when the transposition on the left visual hemifield was attended $\left(F_{1,10}=6.87, P<0.05\right)$. However, not only the parieto-occipital but also the left and right frontal regional means (1 and 7 in Fig. 3) exhibited a marked increase in spectral power on the ipsilateral side of the attended motion condition.

Fig. 4 depicts the mean baseline and normalized spectral power for the $35-51-\mathrm{Hz}$ band across subjects for the three time averages prior to motion onset for the left and right parieto-occipital sites. Fig. 5 shows the grand mean spherical spline (Perrin et al., 1989) interpolated topography of spectral power from 35 to $51 \mathrm{~Hz}$ when subjects attented the whole screen (Fig. 5 left) and after

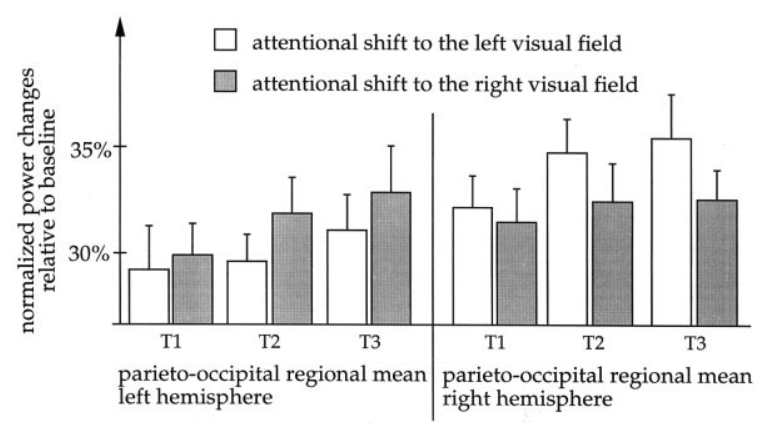

Fig. 4. Time course of grand mean normalized spectral 35-51 $\mathrm{Hz}$ power $(+\mathrm{S}$.E. $)$ relative to baseline for parieto-occipital regional means for stimulus onset (T1: 44-476 ms), arrow onset (T2: 534-956 ms) and stimulus without arrow (T3: 1064-1496 ms) when subjects had to shift their attention to the left (white bars) or right (gray bars) visual hemifield, respectively.

they have shifted their attention to the left visual hemifield (Fig. 5 right). As can be seen in Fig. 4, spectral power increased in the second and third time average at the parieto-occipital regional mean contralateral to the to-be-attended visual hemifield. Consequently, we found a significant regional mean $\times$ attention interaction for time average two $\left(F_{3,30}=4.30, P<0.05\right)$ and three $\left(F_{3,30}\right.$ $=6.46, P<0.01)$ only. Interestingly, ipsilateral parieto-occipital spectral power showed only marginal differences as compared to the first time average in which the whole screen was attended (Figs. 4 and 5).

With respect to the gamma band response extracted from the time domain averaged response
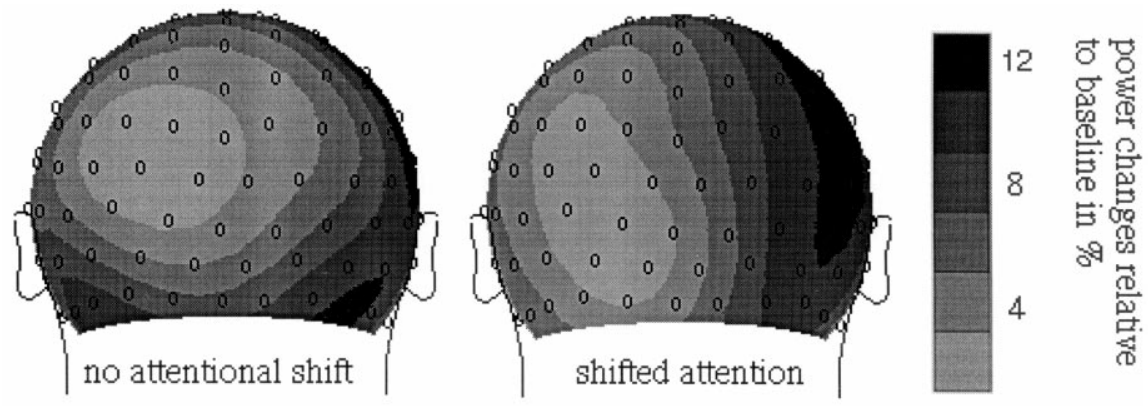

Fig. 5. Spherical spline topographical distributions of the 35-51-Hz band across 11 subjects. Left: $44-476$ ms after stimulus onset, i.e. no attentional shift. Right: 1064-1496 after stimulus onset, i.e. shifted attention (to the left visual hemifield) relative to baseline (black screen). 
(i.e. the ERP), no significant effect could be found in any of the analyses.

\subsubsection{Summary and conclusion}

The present experiment validated the finding of study 1: Power in the gamma band increases with attention to a moving stimulus. No such effect appears in the alpha band range. The enhancement was most prominent at parieto-occipital electrodes contralateral to the to-be-attended visual hemifield. Shifting visual attention to one visual hemifield was related to an increase in spectral power over the contralateral hemisphere with no sign of a power suppression over the ipsilateral hemisphere. Results strongly suggest that induced gamma band activity is closely related to visual information processing and attentional perceptual mechanisms.

\subsubsection{Induced gamma band activity and Gestalt perception}

According to the temporal binding hypothesis, gamma band activity should be prominent when a given stimulus will be perceived as a meaningful object and absent when no such object can be perceived. In the next study we tested this hypothesis by using rotating ambiguous figures on the basis of the law of good figure (gute Gestalt).

\subsection{Study 3}

The following experiment investigated induced gamma band topography and event-related potentials in human EEG associated with perceptual switching associated with the observation of rotating ambiguous figures. The advantage of this procedure is that while the visual input changes continuously (but not its physical parameters like spatial frequency, luminance, etc.), the visual system alternates between two distinct visual experiences perceiving either a happy or sad face.

\subsubsection{Subjects and stimulation}

Participants were shown a rotating bistable figure (1 rev./5 s) that allowed perception of a sad or sunny face depending on orientation and, therefore, was considered to switch at predictable time points when rotated (see Fig. 6). A set of pictures differing in terms of ambiguity and rotation served as control stimuli. A modified version of the Rubin vase that was designed to be symmetrical both in the vertical and the horizontal axis was shown in rotation (see Fig. 6), and several non-ambiguous stimuli containing elements of the figures described above were shown in a stable manner. As an added control, subjects indicated perceptual shifts by means of a key press in a separate condition without EEG-recordings in order to avoid contamination with motor-related potentials and movement artifacts.

Gamma band power was examined in two frequency ranges, $29-45$ and $55-71 \mathrm{~Hz}(45-50 \mathrm{~Hz}$ was omitted to avoid the possibility of $50 \mathrm{~Hz}$ electrical interference). In terms of topography, spectral and ERP measures were grouped into regional means corresponding with sites $\mathrm{Fp} 1, \mathrm{Fp} 2$, $\mathrm{F} 3, \mathrm{~F} 4, \mathrm{~T} 3 / 4, \mathrm{C} 3, \mathrm{C} 4, \mathrm{P} 3, \mathrm{P} 4, \mathrm{O} 1$ and $\mathrm{O} 2$ of the international 10-20 system.

We found greater gamma band EEG activity during the rotation of the faces $\left(F_{1,10}=49.1, P<\right.$ $0.01)$ as compared to the Rubin vase. This enhancement was greatest when both the faces and the vase were in a more meaningful (i.e. vertical as opposed to horizontal $)$ orientation $\left(F_{5,50}=9.4\right.$, $P<0.01)$. Furthermore, gamma band activity did not differ between any of the figures when presented in a stable manner. ERPs were associated with perceptual switching of the sad/happy faces but not with the rotating Rubin vase or the viewing of stable stimuli which supports the proposition that there was no orientation-dependent switching in the Rubin vase condition. The ERP data collected during observation of the rotating sad/happy figure showed a pronounced frontal negative potential. Participants' key presses were shown to display no systematic covariation with the orientation of the stimulus but displayed significant statistical relations with the ERP peak response during the perceptual switch from sad to happy $(r=0.78, P<0.01)$ and from happy to sad ( $r=0.67, P<0.05$; see Fig. 6$)$. This, along with the lack of correlation between ERPs and key press latency during observation of the Rubin vase, supports the use of ERPs to denote perceptual switching. 

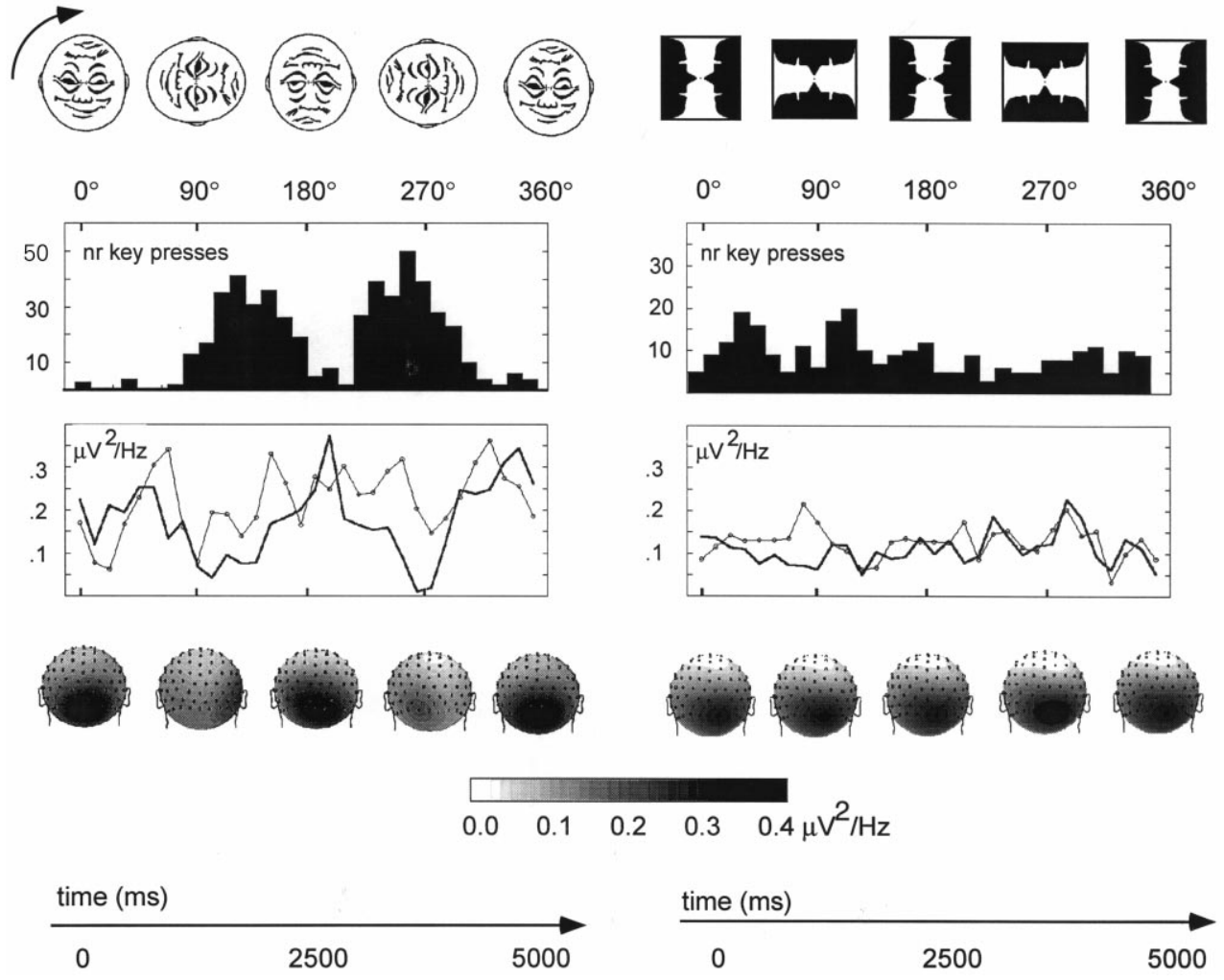

Fig. 6. Grand mean $(N=11)$ behavioral and electrocortical responses to rotating ambiguous stimuli. Left: rotating sad/happy figure. Right: modified Rubin vase. Top line: stimulus orientations attained during one complete rotation $\left(0-360^{\circ}\right)$. Upper panels: histograms of behavioral responses as a function of stimulus orientation. Lower panels: time course of spectral power in the lower (29-45 Hz, bold lines) and higher $(55-71 \mathrm{~Hz}$, thin circled lines) gamma range, obtained at posterior electrodes. Bottom line: back view of the topographical distribution of the spectral power in the $29-45-\mathrm{Hz}$ band during different orientations of the stimuli.

\subsubsection{Summary and conclusion}

The experimental strategy used in this study to isolate perceptual shifts revealed changes in both lower- and higher-frequency gamma band power during vertical orientations of the rotating bistable figures. This effect was most pronounced in the phase-locked switching condition involving the rotating sad/happy stimulus. Although this effect was also seen across recording sites when participants observed the rotating Rubin vase, modulation at posterior electrode sites was found only during rotation of the sad/happy stimulus. A static presentation of the stimuli resulted in overall reduced gamma band power. In summary, the perception of coherently moving stimuli alone was shown to not be the main determinant of the gamma band power modulation. Rather, the re- peated changes between percepts and the associated underlying reorganization appeared to be associated with phasic increases in induced gamma band activity.

\subsubsection{Modulation of hemispheric gamma band activity by processing affective stimuli}

Finally, we examined spectral power in the gamma band while subjects viewed pictures with emotional content. In particular we were interested in whether or not we can find differential hemispheric activation as a function of valence. The valence hypothesis in its more general version would predict the dominance of the right hemisphere for the perception of emotions, regardless of valence (e.g. Davidson 1984; Ehrlichman, 1987; Hirschman and Safer, 1982). No such 
activity is expected when processing neutral pictures.

\subsection{Study 4}

Subjects attended to 60 colored slides from the International Affective System (IAPS; Lang et al., 1997) differing in affective valence and arousal. The set was designed to contain 20 pictures of three affective categories (pleasant, neutral, unpleasant). Each picture was shown in a randomized order for $6 \mathrm{~s}$ with an interstimulus interval between 7 and $12 \mathrm{~s}$. Three blocks of 60 pictures were presented. Three frequency bands in the high-frequency range were obtained using the method by Feige indicated above, and by averaging across the respective frequencies: $\gamma-40=$ $30-50 \mathrm{~Hz}, \gamma-60=50-70 \mathrm{~Hz}$, and $\gamma-80=70-90$ Hz. In addition, the alpha $(8-12 \mathrm{~Hz})$ and beta $(13-19 \mathrm{~Hz})$ bands were analyzed. Each frequency band was baseline corrected by subtracting the mean spectral power of the window prior to picture onset (black screen). Since (1) affective modulation was most likely to be seen over temporal and frontal areas and (2) the signal appeared to be most pronounced at temporal sites upon visual inspection, temporal and frontal electrodes were clustered into regional means according to Fig. 3. Thus, each electrode cluster comprised of seven electrodes.

We found that spectral power for positive and negative valence was most pronounced in the $\gamma-40$ band. In terms of topography, the center of activity in the $\gamma-40$ band for emotional pictures was over the anterior and posterior temporal lobe ventrally to electrodes $\mathrm{F} 3 / \mathrm{F} 4, \mathrm{C} 3 / \mathrm{C} 4$ and $\mathrm{P} 3 / 4$ of the international 10-20 system. In the neutral condition, the center of activity was more on parieto-occipital electrodes, indicating activity related to visual information processing. As can be seen in Fig. 7, there was a strong effect of affective valence in the $\gamma$ - 40 band, with affective pictures showing higher spectral power than neutral pictures.

Most interestingly, a significant hemisphere by valence interaction $\left(F_{1,8}=9.2, \quad P<0.02\right)$ was found which showed that $\gamma-40$ spectral power for pleasant pictures was higher over the right hemi-

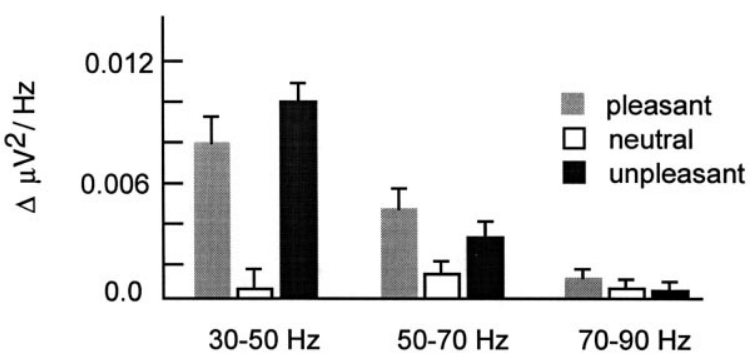

Fig. 7. Grand mean baseline-corrected spectral power in three frequency bands $(30-50,50-70$ and $70-90 \mathrm{~Hz})$ for the affective picture categories, averaged across 128 electrodes.

sphere and power for unpleasant pictures was higher over the left hemisphere. In general, $\gamma-40$ power was significantly higher on right hemisphere temporal electrodes as compared to corresponding electrodes over the left hemisphere $\left(F_{1,8}\right.$ $=19.7, P<0.003$ ).

No effects were found for $\gamma-60(50-70 \mathrm{~Hz})$ and $\gamma-80(70-90 \mathrm{~Hz})$, the alpha and beta bands. In addition, previous findings on frontal asymmetry of EEG alpha measures (for an overview see Davidson and Hugdahl, 1995) could not be replicated using the method presented here.

\subsubsection{Summary and conclusion}

One main result of this experiment is that the processing of emotional pictures induces topographically-specific cortical activity in EEG gamma from 30 to $50 \mathrm{~Hz}$ at temporal and frontal right hemisphere electrode sites. This finding is consistent with the organization of affective perception in widespread networks as stated in our hypothesis. Moreover, the structures involved in these networks may use a specific temporal-spatial pattern of activity to encode the respective emotional valence or arousal associated with external stimuli. The present results are supportive of the idea that cortical structures, which are related to processing of emotional material, coordinate their activity by high-frequency oscillations.

\section{General discussion}

In the present paper we presented four studies 
which aimed at further demonstrating that induced gamma band activity in the human brain is linked to visual perceptual mechanisms and information processing. First, we showed that induced gamma band activity is modulated by spatial selective visual attention. The increase in spectral power when a subject attended to a certain stimulus as compared to when the same stimulus was ignored was replicated in the follow-up study. Thus, the results are in line with our hypothesis based on VEP findings, which consistently show an attentional amplification of certain VEP components (Anllo-Vento et al., 1998; Hillyard and Anllo-Vento, 1998; Hillyard et al., 1998; Luck and Ford, 1998; Mangun, 1995; Mangun et al., 1993). In addition, we have shown that cortical activity in the gamma band range shifted to the contralateral hemisphere when subjects shifted their attention to the left or right visual hemifield. However, we found no sign of a reduction in spectral power on ipsilateral posterior electrodes. These findings are in accordance with a recent study which reported a cortical facilitation during cued shifts of spatial attention using the steady-state visual evoked potential (SSVEP; Müller et al., 1998). When two flickering stimuli were presented in the left and right visual hemifield, the shift of attention to one of the stimuli resulted in an increase of SSVEP amplitude for the attended side with no signs of a SSVEP amplitude reduction for the ignored stimulation side.

In the following experiment, we demonstrated an increase in spectral gamma power on posterior electrode locations when objects were formed by the Gestalt law of good figure (gute Gestalt). Continuous feature changes of the drawings, which were produced by permanent rotation, were not linked with marked increases in the EEG spectral gamma power as compared to baseline. Only when the figure was in or close to a horizontal orientation occipital gamma band power was increased. Thus, our experiment is in line with other studies in which Gestalt principles were used to form an object, such as co-linearity (Tallon et al., 1995; Tallon-Baudry et al., 1996, 1997b; see also this volume), common motion (Lutzenberger et al., 1995) or schematic presentations of faces (Rodriguez et al., 1999). In addition, in- creased induced gamma band power was recently found to be linked to visual recognition of nonstable postures (Slobounov, et al., 2000). Although the center frequencies varied in all of these studies, a common feature is the main activity at occipital electrode locations. In addition, those studies which presented the time by frequency analysis consistently found the peak amplitude in a latency range between 180 to $300 \mathrm{~ms}$ (see also Tallon-Baudry and Bertrand, 1999). Although Pulvermüller et al. (1999) questioned the significance of induced gamma band responses as an index of perceptual processes due to the long latency, it should be mentioned, however, that feature related cortical activity in the VEP is approximately in the same latency range (AnlloVento and Hillyard, 1996; Anllo-Vento et al., 1998; Hillyard and Münte, 1984).

Finally, we have presented evidence that processing of affective pictures is not restricted to activity in posterior areas but also induces activity in temporal and frontal areas. However, when neutral pictures were presented, gamma band activity was most pronounced at parieto-occipital electrodes (Müller et al., 1999). We consider this to be an indicator of the activity of wide spread cell assemblies with members in subcortical (limbic) and neocortical structures related to the processing of emotional material as suggested by several authors (Damasio, 1998; Davidson, 1995, 1998; Davidson and Hugdahl, 1995; De Pascalis, 1989; Derryberry and Tucker, 1992; Lang et al., 1998; LeDoux, 1995a; Liotti and Tucker, 1995; Talbot et al., 1995; Tucker, 1984; Tucker and Dawson, 1984). The bio-informational view of emotions was already proposed by Peter Lang in 1977. He stated that emotions are represented in conceptual networks of propositionally coded information consisting of response, stimulus and meaning concepts (Lang, 1977, 1979). Whether or not gamma band activity reflects the activity of such a network remains speculative at present and is subject to future research. Contrary to previous experiments which focused on alpha desynchronization (see Davidson, 1995, for an overview), we found no effect with respect to the alpha band. Alpha desynchronization seems to be a poor predictor for measuring the activity of 
cortical networks, probably because topographical distribution of alpha and high frequency responses are not inversely related in a one-by-one fashion (Müller, 1998; Müller et al., 1996, 1997b; Tallon-Baudry et al., 1998). Ray and Cole (1985), for instance, reported higher beta activity (16-24 $\mathrm{Hz}$ ) in the right temporal and parietal areas, whereas no effect was found in the alpha band to reflect emotional processes. Furthermore, Aftanas et al. (1998) have found that their measurement for non-linear dynamical coupling of different brain areas distinguished between positive and negative valence for higher frequencies (up to $28 \mathrm{~Hz}$ ) but not for the alpha band.

For the discussion of the influence of possible sources of artifacts like muscle activity and harmonics of lower frequency bands we refer to Gruber et al. (1999), Keil et al. (1999), Müller (1998), Müller et al. (1996, 1997b, 1999), and Pulvermüller et al. (1997). We would like to mention just one methodological issue that refers to the interpretation of topographical distributions in particular with respect to studies 2-4 in which 128 channels montages were used. The problem is the selection of the EEG reference for analysis. In our studies we arithmetically transformed the $\mathrm{Cz}$ recording reference to the average reference prior to spectral analysis. As with every choice of a non-active reference, the question arises as to whether our findings with respect to a given electrode, reflect activity of the tissue below that electrode or result from potentials variations of the reference. For example, it has been argued that the average reference may produce so-called 'ghost fields' (Desmedt et al., 1990) and can produce distortions of focal features (Tomberg et al., 1990) when applied to time-locked signals. It seems likely that this is also possible with induced responses.

One possibility of addressing this problem is the calculation of the current source density (CSD; Nunez, 1990; Perrin et al., 1989) before the transformation into the frequency domain (see experiment 1). This procedure has the advantage that it provides a reference-free measure of electrocortical activity (Lutzenberger et al., 1995). However, when extracting information from CSD one generally emphasizes sharp features in the scalp topography and then dismisses deep and distributed sources (Dien, 1998). In addition, calculating the CSD for single trials may also overestimate high-frequency noise (Law et al., 1993; Biggins et al., 1991) which presents a problem for spectral analyses in particular.

A further approach for addressing undesired effects of the average reference would be the application of distributed source modeling techniques such as the so-called minimum norm (MN) method (Hämäläinen and Ilmoniemi, 1984; Hauk et al., 1998) on the level of single trials. This method is designed to overcome problems associated with an overestimation of focal source configurations. In addition, it is conservative with respect to the effects of noise. We are currently evaluating these algorithms using simulated and real data, comparing average reference, scalp current density and $\mathrm{MN}$ estimates. Although preliminary results suggest that the differences between the methods are substantial, timing and topography of pronounced events in the gamma range appear to be robust with respect to method of data transformation. This position is consistent with the suggestions of Dien (1998). He argued that problems with the average reference may mainly occur when using sparse electrode arrays and, therefore, he recommended using the average reference for deep or distributed sources in combination with high-density electrode arrays.

Results reported to date, including our own, do not provide direct empirical evidence for the temporal binding hypothesis (as the most popular hypothesis about induced gamma band activity). This is partly due to the experimental design, but more so due to the fact that macroscopical recordings like EEG and MEG even with high density electrodes or sensor arrays, do not allow for spatial separation of neighboring cortical areas (e.g. V4 and V5) and thus the synchronization of neural mass activity between them. It was suggested recently that EEG coherency analyses could address this question (Miltner et al., 1999; Rodriguez et al., 1999). In these studies gamma band coherence was calculated for surface electrodes. Greater coherence between a pair or a set of electrodes was interpreted as an indicator of neural synchronization between two areas. How- 
ever, as with all EEG analyses techniques, coherency analysis is confounded with the inverse problem. Theoretically, it is possible that a single alternating dipole causes coherence because of volume conduction. At present, multi unit recordings in visual areas of alert animals seem to be a more promising method of empirically testing the temporal binding hypothesis by showing synchronized neural activity in visual areas which are highly specialized for the processing of the relevant features of a stimulus. A red moving bar should elicit synchronized activity in V1, V4 and V5. As far as we know such evidence is still not yet available. Until presently it seems very probable that induced gamma band oscillations are related and play an important role in perceptual information processing. Whether their functional significance is related to feature binding, scene segmentation, sensory integration, stimulus representation, memory or computational processes or other temporal coding tasks or a combination of some of these processes is subject to future experiments.

\section{Acknowledgements}

We would like to thank Eva Bonna, Ursula Lommen, Heidi Messmer, Klaus Lang and Jürgen Wolf for help in data acquisition and Lisa Green for editorial support. We also thank Prof. Peter Lang, University of Florida, for help in selecting the picture set of study 4 and Prof. Thomas Elbert, University of Konstanz, for constructive discussions. Research was supported by grants from the Deutsche Forschungsgemeinschaft and the Human Frontier Science Program.

\section{References}

Abeles, M, 1982. Local Cortical Circuits. Springer, Berlin.

Aftanas, L.I., Lotova, N.V., Koshkarov, V.I., Makhnev, V.P., Mordvintsev, Y.N., Popov, S.A., 1998. Non-linear dynamic complexity of the human EEG during evoked emotions. Int. J. Psychophysiol. 28, 63-76.

Anllo-Vento, L., Hillyard, S.A., 1996. Selective attention to the color and direction of moving stimuli: electropysiological correlates of hierarchical feature selection. Percept. Psychophys. 58, 191-206.
Anllo-Vento, L., Luck, S.J., Hillyard, S.A., 1998. Spatio-temporal dynamics of attention to color: evidence from human electrophysiology. Hum. Brain Mapp. 6, 216-238.

Attneave, F., 1971. Multistability in perception. Sci. Am. 225, 63-71.

Beauchamp, M.S., Cox, R.W., DeYoe, E.A., 1997. Graded effects of spatial and featural attention on human area MT and associated motion processing areas. J. Neurophysiol. 78, 516-520.

Biggins, C.A., Fein, G., Raz, J., Amir, A., 1991. Artifactually high coherences result from using spherical spline computation of scalp current density. Electroencephalogr. Clin. Neurophysiol. 79, 413-419.

Corbetta, M., Miezin, F.M., Shulman, G.L., Petersen, S.E., 1993. A PET study of visuospatial attention. J. Neurosci. 13, 1202-1226.

Corbetta, M., Shulman, G.L., Miezin, F.M., Petersen, S.E., 1995. Superior parietal cortex activation during spatial attention shifts and visual feature conjunction. Science 270 , 802-805.

Damasio, A.R., 1995. On some functions of the human prefrontal cortex. Ann. N.Y. Acad. Sci. 769, 241-251.

Damasio, A.R., 1998. Emotion in the perspective of an integrated nervous system. Brain Res. Rev. 26, 83-86.

Davidson, R.J., 1984. Affect, cognition, and hemispheric specialization. In: Izard, C.E., Kagan, J, Zajonc, R (Eds.), Emotions, Cognition, and Behavior. Cambridge Press, Cambridge, England, pp. 320-365.

Davidson, R.J., 1992. Anterior cerebral asymmetry and the nature of emotion. Brain Cogn. 20, 125-151.

Davidson, R.J., 1995. Cerebral Asymmetry, Emotion, and Affective Style. MIT Press, Cambridge, MA.

Davidson, R.J., 1998. Anterior electrophysiological asymmetries, emotion, and depression: conceptual and methodological conundrums. Psychophysiology 35, 607-614.

Davidson, R.J., Ekman, P., Saron, C.D., Senulis, J.A., Friesen, W.V., 1990. Approach-withdrawal and cerebral asymmetry: emotional expression and brain physiology. Int. J. Pers. Soc. Psychol. 58, 330-341.

Davidson, R.J., Hugdahl, K., 1995. Brain Asymmetry. MIT Press, Cambridge, MA.

Derryberry, D., Tucker, D.M., 1992. Neural mechanisms of emotion. J. Consult. Clin. Psychol. 60, 329-338.

Desmedt, J.E., Chalkin, V., Tomberg, C., 1990. Emulation of somatosensory evoked potential (SEP) components with the 3-shell head model and the problem of 'ghost potential fields' when using an average reference in brain mapping. Electroencephalogr. Clin. Neurophysiol. 77, 243-258.

Dien, J., 1998. Issues in the application of the average reference: review, critiques, and recommendations. Behav. Res. Inst. Comp. 30, 34-43.

Dien, J., Tucker, D.M., Potts, G., Harty-Speiser, A., 1997. Localization of auditory evoked potentials related to selective intermodal attention. J. Cogn. Neurosci. 9, 799-823.

De Pascalis, V., 1989. 40-Hz asymmetry during recall of emotional events. Int. J. Psychophsiol. 7, 85-96. 
Eckhorn, R., Reitboeck, H.J., Arndt, M., Dicke, P., 1990. Feature linking via synchronization among distributed assemblies: simulations of results from cat visual cortex. N. Comp. 2, 293-307.

Ehrlichman, H., 1987. Hemispheric asymmetry and positivenegative affect. In: Ottoson, D. (Ed.), Duality and Unity of the Brain. Macmillan, Hempshire, pp. 194-206.

Eulitz, C., Maess, B., Pantev, C., Friederici, A.D., Feige, B., Elbert, T., 1996. Oscillatory neuromagnetic activity induced by language and non-language stimuli. Cogn. Brain Res. 4, 121-132.

Feige, B., 1996. Oscillatory brain activity and its analysis on the basis of MEG and EEG. Dissertation Westfälische Wilhelmsuniversität Münster.

Freeman, W.J., 1975. Mass Action in the Nervous System. Academic Press, New York.

Freeman, W.J., 1996. Feedback models of gamma rhythms. TINS 19, 468.

Freeman, W.J., van Dijk, B.W, 1987. Spatial patterns of visual cortical fast EEG during conditioned reflex in a rhesus monkey. Brain Res. 422, 267-276.

Freeman, W.J., di Prisco, G.V., 1986. EEG spatial pattern differences with discriminated odors manifest chaotic and limit cycle attractors in olfactory bulb of rabbits. In: Palm, G., Aertsen, A. (Eds.), Brain Theory. Springer, Berlin, pp. 97-119.

Galambos, R., 1992. A comparison of certain gamma band $(40-\mathrm{Hz})$ brain rhythms in cat and man. In: Basar, E., Bullock, T.H. (Eds.), Induced Rhythms in the Brain. Birkhäuser, Boston, pp. 201-216.

Gray, C.M., Engel, A.K., König, P., Singer, W., 1990. Stimulus-dependent neuronal oscillations in cat visual cortex: receptive field properties and feature dependence. Eur. J. Neurosci. 2, 607-619.

Gray, C.M., König, P., Engel, A.K., Singer, W., 1989. Oscillatory responses in cat visual cortex exhibit inter-columnar synchronization which reflects global stimulus properties. Nature 338, 334-337.

Gray, C.M., Singer, W., 1989. Stimulus-specific neuronal oscillations in orientation columns of cat visual cortex. Proc. Nat. Acad. Sci. USA 86, 1698-1702.

Gray, C.M., Singer, W., 1987. Stimulus-specific neuronal oscillations in cat visual cortex: a cortical functional unit. Soc. Neurosci. Abstr. 13, 404.3.

Gruber, T., Müller, M.M., Keil, A., Elbert, T., 1999. Selective visual-spatial attention alters induced gamma band responses in the human EEG. Clin. Neurophysiol. 10, 2074-2085.

Hari, R., Salmelin, R., 1997. Human cortical oscillations: a neuromagnetic view through the scull. TINS 20, 44-49.

Hämäläinen, M., Ilmoniemi, R., 1984. Interpreting Measured Magnetic Fields of the Brain: Estimates of Current Distributions. Helsinki University of Technology, Helsinki.

Haug, A.B., Baudewig, J., Paulus, W., 1998. Selective activation of human cortical area V5A by a rotating visual stimulus in fMRI; implication of attentional mechanisms. NeuroReport 9, 611-614.
Hauk, O., Berg, P., Wienbruch, C., Rockstroh, B., Elbert, T., 1998. The minimum norm method as an effective mapping tool for MEG analysis. Biomag98. Sendai, Japan.

Heinze, H.J., Mangun, G.R., Burchert, W. et al., 1994. Combined spatial and temporal imaging of brain activity during visual selective attention in humans. Nature 372, 543-546.

Herrmann, S., Mecklinger, A., Pfeifer, E., 1999. Gamma responses and ERPs in a visual classification task. Clin. Neurophysiol. 110, 636-642.

Hillyard, S.A., Anllo-Vento, L., 1998. Event-related brain potentials in the study of visual selective attention. Proc. Nat. Acad. Sci. USA 95, 781-787.

Hillyard, S.A., Münte, T.F., 1984. Selective attention to color and location: an analysis with event-related brain potentials. Percept. Psychophys. 36, 185-198.

Hillyard, S.A., Vogel, E.K., Luck, S.J., 1998. Sensory gain control (amplification) as a mechanism of selective attention: electrophysiological and neuroimaging evidence. Philos. Trans. R. Soc. Ser. B 353, 1257-1270.

Hirschman, R.S., Safer, M.A., 1982. Hemisphere differences in perceiving positive and negative emotions. Cortex 18, 569-580.

Jokeit, H., Makeig, S., 1994. Different event-related patterns of gamma-band power in brain waves of fast- and slow-reacting subjects. Proc. Nat. Acad. Sci. USA 91, 6339-6343.

Junghöfer, M., Elbert, T., Tucker, D.M., Rockstroh, B.S., 2000. Statistical control of artifacts in dense array EEG/MEG Studies. Psychophysiology 37, 523-532.

Kanizsa, G., Luccio, R., 1995. Multistability as a research tool in experimental phenomenology. In: Kruse, P., Stadler, M. (Eds.), Ambiguity in Mind and Nature. Springer, Berlin, pp. 47-68.

Keil, A., Müller, M.M., Ray, W.J., Elbert, T., Gruber, T., 1999. Human gamma band activity and perception of a gestalt. J. Neurosci. 19, 7152-7161.

Kristeva-Feige, R., Feige, B., Makeig, S., Ross, B., Elbert, T., 1993. Oscillatory brain activity during a motor task. NeuroReport 4, 1291-1294.

LaBerge, D., 1995. Attentional Processing. Harvard University Press, Cambridge.

Lang, P.J., 1977. Imagery in therapy: an information processing analysis of fear. Behav. Ther. 8, 862-886.

Lang, P.J., 1979. A bio-informational theory of emotional imagery. Psychophysiology 16, 495-512.

Lang, P.J., Bradley, M.M., Cuthbert, B.N., 1997. International Affective Picture System (IAPS): Technical Manual and Affective Ratings. University of Florida. The Center for Research in Psychophysiology, Gainesville.

Lang, P.J., Bradley, M.M., Fitzsimmons, J.R. et al., 1998. Emotional arousal and activation of the visual cortex: an fMRI analysis. Psychophysiology 35, 199-210.

Law, S.K., Rohrbaugh, J.W., Adams, C.M., Eckhart, M.J., 1993. Improving spatial and temporal resolution in evoked EEG responses using surface Laplacians. Electroencephalogr. Clin. Neurophysiol. 88, 309-322. 
LeDoux, J.E., 1995a. Emotion: clues from the brain. Annu. Rev. Psychol. 46, 209-235.

LeDoux, J.E., 1995b. In search of an emotional system in the brain: leaping from fear to emotion and consciousness. In: Gazzaniga, M.S. (Ed.), The Cognitive Neurosciences. MIT Press, Cambridge, pp. 1049-1061.

Liotti, M., Tucker, D.M., 1995. Emotion in asymmetric corticolimbic networks. In: Davidson, R.J., Hughdahl, K. (Eds.), Brain Asymmetry. MIT Press, Cambridge, pp. 389-423.

Luck, S.J., Ford, M.A., 1998. On the role of selective attention in visual perception. Proc. Nat. Acad. Sci. USA 95, 825-830.

Lutzenberger, W., Pulvermüller, F., Birbaumer, N., 1994. Words and pseudowords elicit distinct patterns of $30-\mathrm{Hz}$ EEG responses in humans. Neurosci. Lett. 176, 115-118.

Lutzenberger, W., Pulvermüller, F., Elbert, T., Birbaumer, N., 1995. Visual stimulation alters local $40-\mathrm{Hz}$ responses in humans: an EEG-study. Neurosci. Lett. 183, 39-42.

Maier, J., Dagneli, H., Spekreijse, H., van Dijk, B.W., 1987. Principal component analysis for source localization of VEPs in man. Vis. Res. 27, 165-177.

Makeig, S., 1993. Auditory event-related dynamics of the EEG spectrum and effects of exposure to tones. Electroencephogr. Clin. Neurophysiol. 86, 283-293.

Malsburg, C.v.d., Schneider, W., 1986. A neural cocktail-party processor. Biol. Cyber. 54, 29-40.

Mangun, G.R., 1995. Neural mechanisms of visual selective attention. Psychophysiology 32, 4-18.

Mangun, G.R., Hillyard, S.A., Luck, S.J., 1993. Electrocortical substrates of visual selective attention. In: Gazzaniga, M.S. (Ed.), The Cognitive Neurosciences. MIT Press, Cambridge, pp. 219-243.

Mangun, G.R., Hopfinger, J.B., Kussmaul, C.L., Fletcher, E.M., Heinze, H.J., 1997. Covariations in ERP and PET measures of spatial selective attention in human extrastriate visual cortex. Hum. Brain Mapp. 5, 273-279.

Martinez, A., Anllo-Vento, L., Sereno, M.I. et al., 1999. Involvement of striate and extrastriate visual cortical areas in spatial attention. Nat. Neurosci. 2, 364-369.

McCarthy, G., Wood, C.C., 1985. Scalp distributions of eventrelated potentials: an ambiguity associated with analysis of variance models. Electroencephogr. Clin. Neurophysiol. 62, 203-208.

Milner, P.M., 1974. A model for visual shape recognition. Psychol. Rev. 81, 521-535.

Miltner, W.H.R., Braun, C., Arnold, M., Witte, H., Taub, E., 1999. Coherence of gamma-band activity as a basis for associative learning. Nature 397, 434-436.

Müller, M.M., 1998. Oscillatory cortical activities in the human brain. Habilitation, Universität Konstanz.

Müller, M.M., Bosch, J., Elbert, T. et al., 1996. Visually induced gamma-band responses in human electroencephalographic activity - a link to animal studies. Exp. Brain Res. 112, 96-102.

Müller, M.M., Elbert, T., Rockstroh, B., 1997a. Visuell induzierte gammabandaktivität im menschlichen EEGausdruck corticaler reizrepräsentation? Z. Exp. Psy. 44, 186-212.
Müller, M.M., Junghöfer, M., Elbert, T., Rockstroh, B., $1997 \mathrm{~b}$. Visually induced gamma-band responses to coherent and incoherent motion: a replication study. NeuroReport 8, 2575-2579.

Müller, M.M., Keil, T., Gruber, T., Elbert, T., 1999. Processing of affective pictures modulates right-hemispheric gamma band EEG activity. Clin. Neurophysiol. 110, 1913-1920.

Müller, M.M., Teder-Sälejärvi, W., Hillyard, S.A., 1998. The time course of cortical facilitation during cued shifts of spatial attention. Nat. Neurosci. 1, 631-634.

Nunez, P.L., 1990. Localization of Brain Activity with Electroencephalography. Raven Press, New York.

Ota, T., Toyoshima, R., Yamauchi, T., 1996. Measurements by biphasic changes of the alpha band amplitude as indicators of arousal level. Int. J. Psychophysiol. 24, 25-37.

Perrin, F., Pernier, J., Bertrand, O., Echallier, J.F., 1989. Spherical splines for scalp potential and current source density mapping. Electroencephogr. Clin. Neurophysiol. 72, 184-187.

Pfurtscheller, G., 1992. Event-related synchronization (ERS): an electrophysiological correlate of cortical areas at rest. Electroencephogr. Clin. Neurophysiol. 83, 62-69.

Pfurtscheller, G., Aranibar, A., 1979. Evaluation of event-related desynchronization (ERD) preceding and following voluntary self-paced movement. Electroencephogr. Clin. Neurophysiol. 46, 138-146.

Pfurtscheller, G., Flotzinger, D., Kalcher, J., 1993. Brain-computer interface - a new communication device for handicapped persons. J. Microcomp. Appl. 16, 293-299.

Pfurtscheller, G., Klimesch, W., 1992. Event-related synchronization and desynchronization of alpha and beta waves in a cognitive task. In: Basar, E., Bullock, T.H. (Eds.), Induced Rhythms in the Brain. Birkhäuser, Boston, pp. 117-128.

Posner, I.P., Petersen, S.E., 1990. The attention system of the human brain. Annu. Rev. Neurosci. 13, 25-42.

Posner, M.I., Dehaene, S., 1994. Attentional networks. TINS 17, 75-79.

Priestley, M.B., 1988. Non-Linear and Non-Stationary Time Series Analysis. London, Academic Press.

Pulvermüller, F., Birbaumer, N., Lutzenberger, W., Mohr, B., 1997. High-frequency brain activity: its possible role in attention, perception and language processing. Prog. Neurobiol. 52, 427-444.

Pulvermüller, F., Eulitz, C., Pantev, C. et al., 1996. Highfrequency cortical responses reflect lexical processing: a MEG study. Electroencephogr. Clin. Neurophysiol. 98, $76-85$.

Pulvermüller, F., Keil, A., Elbert, T., 1999. High-frequency brain activity: perception or active memory? TICS 3, 250-253.

Pulvermüller, F., Lutzenberger, W., Preissl, H., Birbaumer, N., 1995. Spectral responses in the gamma-band: physiological signs of higher cognitive processes. NeuroReport 6, 2059-2064.

Qian, S., Chen, D., 1993. Discete gabor transform. IEEE Trans. Sig. Proc. 41, 2429-2438. 
Ray, W.J., Cole, H.W., 1985. EEG alpha activity reflects attentional demands, and beta activity reflects emotional and cognitive processes. Science 228, 750-752.

Rodriguez, E., George, N., Lachaux, J.P., Martinerie, J., Renault, B., Varela, F.J., 1999. Perception's shadow: long-distance synchronization of human brain activity. Nature 397, 430-433.

Singer, W., Gray, C.M., 1995. Visual feature integration and the temporal correlation hypothesis. Ann. Rev. Neurosci. 18, 555-586.

Slobounov, S., Tutwiler, R., Slobounova, E., Rearick, M., Ray, W., 2000. Human oscillatory brain activity within gamma band $(30-50 \mathrm{~Hz})$ induced by visual recognition of non-stable postures. Cogn. Brain Res. 9, 177-192.

Talbot, J.D., Villemure, J.G., Bushnell, M.C., Duncan, G.H., 1995. Evaluation of pain perception after anterior capsulotomy: a case report. Somatosens. Mot. Res. 12, 115-126.

Tallon, C., Bertrand, O., Bouchet, P., Pernier, J., 1995. Gamma-range activity evoked by coherent visual stimuli in humans. Eur. J. Neurosci. 7, 1285-1291.

Tallon-Baudry, C., Bertrand, O., 1999. Oscillatory gamma activity in humans and its role in object representation. TICS 3, 151-162.

Tallon-Baudry, C., Bertrand, O., Delpuech, C., Pernier, J.,
1996. Stimulus specificity of phase-locked and non-phaselocked $40 \mathrm{~Hz}$ visual response in human. J. Neurosci. 16, 4240-4249.

Tallon-Baudry, C., Bertrand, O., Delpuech, C., Pernier, J., 1997a. Oscillatory gamma-band $(30-70 \mathrm{~Hz})$ activity induced by a visual search task in human. J. Neurosci. 17, 722-734.

Tallon-Baudry, C., Bertrand, O., Wienbruch, C., Ross, B., Pantev, C., 1997b. Combined EEG and MEG recordings of visual $40-\mathrm{Hz}$ responses to illusory triangles in human. NeuroReport 8, 1103-1107.

Tallon-Baudry, C., Bertrand, O., Peronnet, F., Pernier, J., 1998. Induced gamma-band activity during the delay of a visual short-term memory task in humans. J. Neurosci. 18, 4244-4254.

Tomberg, C., Noel, P., Ozaki, I., Desmedt, J.E., 1990. Inadequacy of the average reference for the topographic mapping of focal enhancements of brain potentials. Electroencephogr. Clin. Neurophysiol. 77, 259-265.

Tucker, D.M., 1984. Lateral brain function in normal and disordered emotion: interpreting electroencephalographic evidence. Biol. Psychol. 19, 219-235.

Tucker, D.M., Dawson, S.L., 1984. Asymmetric EEG changes as method actors generated emotions. Biol. Psychol. 19, $63-75$. 